\title{
Dual Quaternion Sample Reduction for SE(2) Estimation
}

\author{
Kailai Li, Florian Pfaff, and Uwe D. Hanebeck \\ Intelligent Sensor-Actuator-Systems Laboratory (ISAS) \\ Institute for Anthropomatics and Robotics \\ Karlsruhe Institute of Technology (KIT), Germany \\ kailai.li@kit.edu, florian.pfaff@kit.edu, uwe.hanebeck@kit.edu
}

\begin{abstract}
We present a novel sample reduction scheme for random variables belonging to the $S E(2)$ group by means of Dirac mixture approximation. For this, dual quaternions are employed to represent uncertain planar transformations. The Cramér-von Mises distance is modified as a smooth metric to measure the statistical distance between Dirac mixtures on the manifold of planar dual quaternions. Samples of reduced size are then obtained by minimizing the probability divergence via Riemannian optimization while interpreting the correlation between rotation and translation. We further deploy the proposed scheme for nonparametric modeling of estimates for nonlinear SE(2) estimation. Simulations show superior tracking performance of the sample reduction-based filter compared with Monte Carlo-based as well as parametric model-based planar dual quaternion filters.
\end{abstract}

\section{INTRODUCTION}

Estimation of planar motions is ubiquitous and play a fundamental role in many application scenarios, such as odometry and scene reconstruction, object tracking as well as remote sensing, etc [1]-[5]. Planar motions, incorporating both rotations and translations on a plane, are mathematically described by elements belonging to the two-dimensional special Euclidean group $\mathrm{SE}(2)$. Conventionally, elements of the $\mathrm{SE}(2)$ group are parameterized by the $3 \times 3$ homogeneous matrices. However, with nine elements used to represent the three DoF, they contain a large degree of redundancy, resulting in numerical instabilities and memory inefficiencies. Dual quaternions, defined as $\mathbf{x}=\mathbf{x}_{\mathrm{r}}+\epsilon \mathbf{x}_{\mathrm{s}}$, combine quaternion and dual number theory. The real part $x_{r}$ is a quaternion representing the rotation and $\mathrm{x}_{\mathrm{S}}$ denotes the dual part encoding the translation. $\epsilon$ is the dual unit and satisfies $\epsilon^{2}=0$. In contrast to matrix representations, dual quaternions can parameterize planar motions in the form of four-dimensional vectors with only one degree of redundancy. Conciser ways are thereby enabled for modeling the uncertainty and recursive estimation of planar transformations.

Therefore, we employ planar dual quaternions to parameterize SE(2) states in this paper. Due to the underlying nonlinear group structure, stochastic modeling of uncertain $\mathrm{SE}(2)$ states is usually done in a locally linearized space using Gaussian distributions [6]. Nonlinear estimation approaches can then be proposed by using popular Bayesian filtering schemes such as the extended Kalman filter (EKF) [7] or the unscented Kalman filter (UKF) [8]. However, such a probabilistic model is established under the assumption of local perturbations,
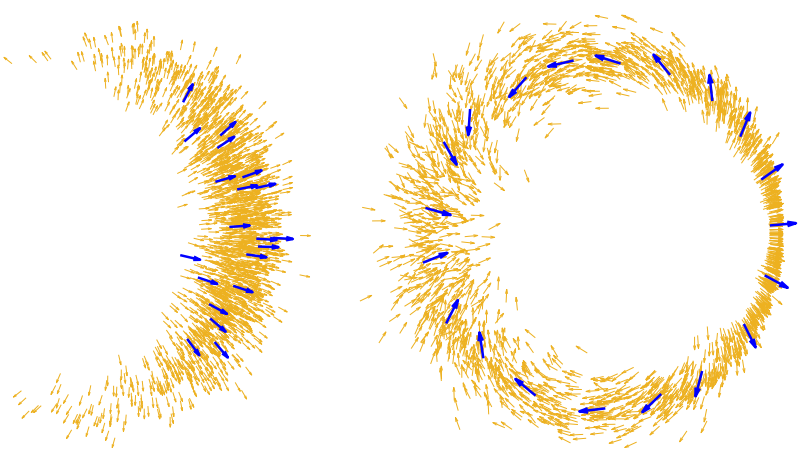

Figure 1: Examples of the proposed sample reduction technique for planar rigid motions. Here, 2000 random samples (yellow) drawn from different underlying distributions are optimally approximated by 20 samples (blue) on the manifold of planar dual quaternions.

which can be easily violated under large noise levels (e.g., due to low-cost sensors or strong nonlinearities).

Dual quaternions representing rigid transformations naturally form a nonlinear manifold and its nonlinearity mainly results from the rotation component. Thus, in order to stochastically model uncertain dual quaternions directly on the manifold without linearization, novel probabilistic density functions (PDFs) have been proposed based on directional statistics. In [9], the Bingham distribution was employed to model the real part of dual quaternions while the translation part was assumed to be Gaussian-distributed. A corresponding filtering scheme was established using the unscented transform [10] and successfully deployed for stereo visual odometry [9]. However, the approach lacks probabilistic interpretation of correlations between the real and dual part, resulting in overoptimistic estimates. Another probabilistic framework, the socalled projected Gaussian distribution (PGD), was introduced in [11] based on hyperspherical geometry. Further, a mixture model was established for addressing multimodality. But its inference scheme requires approximations under the assumption of small rotation uncertainties. In [12], a new distribution was proposed based on the Bingham distribution to directly model uncertain planar dual quaternions while considering correlated real and dual part. Corresponding Bayesian filters [13], [14] 
were established based thereon by exploiting the unscented transform as well as the progressive filtering scheme [15] and were further applied to simultaneous localization and mapping [16].

Though stochastic modeling approaches relying on specific PDFs provide effective solutions for dual quaternion estimation, several shortcomings exist. In practice, the information geometry of the uncertainty is rather arbitrary on the manifold of dual quaternions, meaning parametric forms can be largely violated. Also, there is no closed-form solution for computing the normalization constant of such distributions (e.g., for PGD or Bingham-based distributions). Thus, corresponding filters often rely on samples, generated by either deterministic or Monte Carlo-based sampling schemes, for recursive estimation. The posterior estimate is essentially a re-approximation of the updated samples via model fitting. Though the sampling schemes contribute to handling nonlinear dynamics [8], the refitting step still imposes the parametric form of the underlying distribution.

Sample-based modeling approaches provide an intuitive way to represent arbitrary probability distributions in a nonparametric manner. Components of a Dirac mixture can be located on the samples for discrete probabilistic modeling. Corresponding Bayesian filtering schemes, e.g., the particle filter [17], have been established based thereon for nonlinear estimation. However, naively exploiting empirical samples to represent stochastic dynamics typically suffers from inefficiency issues w.r.t. representativeness and runtime. Also, random samples cannot guarantee reproducible results. Therefore, some works introduced deterministic schemes for nonparametric modeling of estimates in the context of planar motion estimation. In [18], a grid-based approach was proposed on the circular domain for modeling random angular variables and further extended via Rao-Blackwellization for $\operatorname{SE}(2)$ estimation [19]. However, the performance of such schemes depends highly on the grid resolution, since grid points are generated equidistantly on the circle and not adaptive to the shape of the underlying distribution [20]. A high resolution of the grid is usually required for the desired tracking accuracy, resulting in both runtime and memory inefficiencies.

Thus, using fewer but more representative samples is appealing for discrete stochastic modeling. In [21], a sample reduction scheme was established for reducing the number of components of a Dirac mixture by minimizing a multivariate generalization of the Cramér-von Mises distance. For that, the so-called localized cumulative distribution (LCD) of Dirac mixtures was proposed for smoothly characterizing discrete probabilistic models. While this technique has been successfully deployed for Euclidean spaces and was further extended to the spherical domain [22], there exists no counterpart for states belonging to the group of planar rigid body motions.

In this work, we develop an LCD-based sample reduction scheme for random $\mathrm{SE}(2)$ states represented by dual quaternions (see examples in Fig. 1). A geometry-aware metric is proposed for measuring the statistical distance of Dirac mixtures on the manifold of planar dual quaternions. It is established by synthesizing the von Mises and Gaussian kernels that measure the probability mass around the real and dual part, respectively. Components of the approximating Dirac mixture are then located to on-manifold supports minimizing its statistical divergence to the target Dirac mixture by means of Riemannian optimization. The proposed approximation approach is deployed to a sample-based planar dual quaternion filter for nonlinear $\operatorname{SE}(2)$ estimation. As the reduced samples are deterministic and more representative, the novel approach shows improved sample efficiency than a plain particle filter. Also, in contrast to parametric modeling approaches, the proposed scheme enables (approximated) modeling of arbitrary distributions and considers the correlation between the real and dual part.

The remainder of this paper is structured as follows. Preliminaries about the dual quaternion representation of $\mathrm{SE}(2)$ states will be given in Sec. II. The novel sample reduction scheme on the manifold of planar dual quaternions will be proposed in Sec. III. Based thereon, we will give the proposed sample reduction-based $\mathrm{SE}(2)$ estimator in Sec. IV, which is followed by the evaluation in Sec. V. The work will be concluded in Sec. VI.

\section{Preliminaries}

For the sake of conciseness, we formulate dual quaternions parameterizing planar motions into the vector form $\mathbf{x}=\left[\mathbf{x}_{\mathrm{r}}^{\top}, \mathbf{x}_{\mathrm{s}}^{\top}\right]^{\top} \in \mathbb{R}^{4}$, with

$$
\begin{aligned}
& \mathbf{x}_{\mathrm{r}}=[\cos (\theta / 2), \sin (\theta / 2)]^{\top} \in \mathbb{S}^{1}, \\
& \mathbf{x}_{\mathrm{s}}=\frac{1}{2} \mathbf{t} \otimes \mathbf{x}_{\mathrm{r}} \in \mathbb{R}^{2},
\end{aligned}
$$

being the real and dual part, respectively. It denotes a rotation around $z$-axis through angle $\theta$ followed by a translation of $\mathbf{t} \in \mathbb{R}^{2}$ on the $x y$-plane. The real part is formulated as a quaternion for denoting the rotation and the dual part encodes the translation by composing the real part via the Hamilton product $\otimes$. Therefore, planar dual quaternions naturally form a manifold consisting of the circular domain and the twodimensional Euclidean space, i.e., $\mathrm{x} \in \mathbb{S}^{1} \times \mathbb{R}^{2} \subset \mathbb{R}^{4}$.

Aggregating two arbitrary planar dual quaternions can be formulated into a regular matrix-vector multiplication. For instance, $\forall \mathbf{x}=\left[x_{0}, x_{1}, x_{2}, x_{3}\right]^{\top}, \mathbf{y}=\left[y_{0}, y_{1}, y_{2}, y_{3}\right]^{\top} \in$ $\mathbb{S}^{1} \times \mathbb{R}^{2}$, the aggregation $\mathbf{x} \boxplus \mathbf{y}=\mathcal{Q}_{\mathbf{x}}^{\llcorner} \mathbf{y}=\mathcal{Q}_{\mathbf{y}}^{\lrcorner} \mathbf{x}$, with

$$
\mathcal{Q}_{\mathbf{x}}^{\llcorner}=\left[\begin{array}{rrrr}
x_{0} & -x_{1} & 0 & 0 \\
x_{1} & x_{0} & 0 & 0 \\
x_{2} & x_{3} & x_{0} & -x_{1} \\
x_{3} & -x_{2} & x_{1} & x_{0}
\end{array}\right], \mathcal{Q}_{\mathbf{y}}^{\lrcorner}=\left[\begin{array}{rrrr}
y_{0} & -y_{1} & 0 & 0 \\
y_{1} & y_{0} & 0 & 0 \\
y_{2} & -y_{3} & y_{0} & y_{1} \\
y_{3} & y_{2} & -y_{1} & y_{0}
\end{array}\right] .
$$

Any vector $\mathbf{s} \in \mathbb{R}^{2}$ can be transformed by the planar dual quaternion $\mathbf{x} \in \mathbb{S}^{1} \times \mathbb{R}^{2}$ according to

$$
\mathbf{s}^{\prime}=\left(\mathbf{x} \boxplus\left[1,0, \mathbf{s}^{\top}\right]^{\top} \boxplus \mathbf{x}^{\odot}\right)_{3: 4},
$$

with $\mathbf{x}^{\odot}=\left[x_{0},-x_{1},-x_{2},-x_{3}\right]^{\top}$ being the conjugate of $\mathbf{x}$. Here, $\mathbf{s}$ is expressed in the form of a planar dual quaternion and the last two elements of the vector after the operation are taken out to recover the transformed location. A more detailed 
introduction about the arithmetic and manifold structure of dual quaternions can be found in [14], [23].

\section{DUAL QUATERNION-BASED SE(2) SAMPLE REDUCTION}

\section{A. Problem Formulation}

Given a set of random planar dual quaternion samples $\tilde{\mathbb{X}}=$ $\left\{\tilde{\mathbf{x}}_{i}\right\}_{i=1}^{m} \subset \mathbb{S}^{1} \times \mathbb{R}^{2}$, a Dirac mixture is employed to represent its underlying distribution as

$$
f_{\tilde{\mathbf{X}}}(\mathbf{x})=\sum_{i=1}^{m} \tilde{\omega}_{i} \delta\left(\mathbf{x}-\tilde{\mathbf{x}}_{i}\right),
$$

with $\tilde{\omega}_{i}$ being the weights, which satisfy $\sum_{i=1}^{m} \tilde{\omega}_{i}=1$. We aim to approximate the target Dirac mixture above using another Dirac mixture of reduced number of components given by

$$
f_{\mathbb{X}}(\mathbf{x})=\sum_{i=1}^{n} \omega_{i} \delta\left(\mathbf{x}-\mathbf{x}_{i}\right)
$$

Here, $\sum_{i=1}^{n} \omega_{i}=1$ and $\mathbb{X}=\left\{\mathbf{x}_{i}\right\}_{i=1}^{n} \subset \mathbb{S}^{1} \times \mathbb{R}^{2}$ denotes the set of approximating planar dual quaternion samples.

In [21], an optimal sample reduction technique was proposed to approximate multivariate Dirac mixtures in Euclidean spaces. However, this approach cannot be trivially extended to planar dual quaternions due to the underlying nonlinear manifold structure. In the remainder of this section, a geometryaware kernel will be proposed for quantifying the on-manifold probability mass in the sense of the LCD [24]. Based thereon, the Cramér-von Mises distance will be modified as the metric measuring the statistical distance between Dirac mixtures on the manifold of planar dual quaternions.

\section{B. On-Manifold Localized Cumulative Distribution (M-LCD)}

We propose the so-called on-manifold localized cumulative distribution (M-LCD) to quantify the probability mass of a discrete density on a smooth manifold. More specifically, the M-LCD of distribution $g: \mathbb{M} \rightarrow \mathbb{R}_{+}$is defined as

$$
\mathcal{G}(\boldsymbol{\nu}, \tau)=\int_{\mathbb{M}} g(\mathbf{x}) \kappa(\mathbf{x} ; \boldsymbol{\nu}, \tau) \mathrm{d} \mathbf{x},
$$

with $\kappa(\mathbf{x} ; \boldsymbol{\nu}, \tau)$ being the kernel function located at $\boldsymbol{\nu} \in \mathbb{M}$ with a dispersion of $\tau \in \mathbb{R}_{+}$. A smooth kernel is preferable as it leads to simpler optimization problems of maintaining the probability mass for approximation [21]. Consequently, the MLCD of the approximating Dirac mixture in (4) can be derived as

$$
\begin{aligned}
\mathcal{F}_{\mathbb{X}}(\boldsymbol{\nu}, \tau) & =\int_{\mathbb{S}^{1} \times \mathbb{R}^{2}} \sum_{i=1}^{n} \omega_{i} \delta\left(\mathbf{x}-\mathbf{x}_{i}\right) \kappa(\mathbf{x} ; \boldsymbol{\nu}, \tau) \mathrm{d} \mathbf{x} \\
& =\sum_{i=1}^{n} \omega_{i} \kappa\left(\mathbf{x}_{i} ; \boldsymbol{\nu}, \tau\right),
\end{aligned}
$$

with the kernel function being evaluated at each Dirac support $\mathbf{x}_{i} \in \mathbb{S}^{1} \times \mathbb{R}^{2}$. Similarly, the target M-LCD can be derived in the following form

$$
\mathcal{F}_{\tilde{\mathbf{X}}}(\boldsymbol{\nu}, \tau)=\sum_{i=1}^{m} \tilde{\omega}_{i} \kappa\left(\tilde{\mathbf{x}}_{i} ; \boldsymbol{\nu}, \tau\right)
$$

The reason of employing such a cumulative distribution for defining the statistical distance lies in the fact that two Dirac mixtures do not share common supports, thus cannot be compared directly. The standard cumulative distribution is generally asymmetric and only defined for scalar PDFs. Therefore, the localized cumulative distribution was established in [24] for PDFs in higher-dimensional spaces by incorporating the kernel function.

Synthesizing a smooth kernel function for dual quaternion sample reduction is non-trivial. Since the manifold $\mathbb{S}^{1} \times \mathbb{R}^{2}$ exhibits a nonlinear and partially periodic geometric structure, the kernel function should be designed in a geometry-aware manner. For that, we define an isotropic and separable kernel in the form of $\kappa(\mathbf{x} ; \boldsymbol{\nu}, \tau)=\kappa_{\mathrm{r}}\left(\mathbf{x}_{\mathrm{r}} ; \boldsymbol{\nu}_{\mathrm{r}}, \tau\right) \kappa_{\mathrm{s}}\left(\mathbf{x}_{\mathrm{s}} ; \boldsymbol{\nu}_{\mathrm{s}}, \tau\right)$, with

$$
\begin{aligned}
& \kappa_{\mathrm{r}}\left(\mathbf{x}_{\mathrm{r}} ; \boldsymbol{\nu}_{\mathrm{r}}, \tau\right)=\exp \left(\tau \boldsymbol{\nu}_{\mathrm{r}}^{\top} \mathbf{x}_{\mathrm{r}}\right) \\
& \kappa_{\mathrm{s}}\left(\mathbf{x}_{\mathrm{s}} ; \boldsymbol{\nu}_{\mathrm{s}}, \tau\right)=\exp \left(-\tau\left(\mathbf{x}_{\mathrm{s}}-\boldsymbol{\nu}_{\mathrm{s}}\right)^{\top}\left(\mathbf{x}_{\mathrm{s}}-\boldsymbol{\nu}_{\mathrm{s}}\right)\right)
\end{aligned}
$$

measuring the uncertainty at $\mathbf{x}=\left[\mathbf{x}_{\mathrm{r}}^{\top}, \mathbf{x}_{\mathrm{s}}^{\top}\right]^{\top} \in \mathbb{S}^{1} \times \mathbb{R}^{2}$. The kernel consists of a von Mises-like component centered at $\nu_{\mathrm{r}} \in \mathbb{S}^{1}$ evaluating the real part and a Gaussian-like component at $\boldsymbol{\nu}_{\mathrm{s}} \in \mathbb{R}^{2}$ the dual part. The two kernel components share the dispersion parameter $\tau$. Thus, uncertainties can be quantified adaptively to the manifold structure. Further, the shared dispersion parameter $\tau$ allows considering the dependency between the real and dual part. We will further show in the next subsection that such a synthesis also guarantees a unique and closed-form solution for computing the Cramér-von Mises distance in the M-LCD sense.

\section{Statistical Distance of Dirac Mixtures on the Manifold of Planar Dual Quaternions}

We further propose the M-LCD-based Cramér-von Mises distance to measure the statistical divergence between Dirac mixtures at the approximating set $\mathbb{X}$ and the target set $\tilde{\mathbb{X}}$. It is defined as

$$
\mathcal{D}(\mathbb{X})=\int_{\mathbb{R}_{+}} \mathcal{W}(\tau) \int_{\mathbb{S}^{1} \times \mathbb{R}^{2}}\left(\mathcal{F}_{\mathbb{X}}(\boldsymbol{\nu}, \tau)-\mathcal{F}_{\tilde{\mathbb{X}}}(\boldsymbol{\nu}, \tau)\right)^{2} \mathrm{~d} \boldsymbol{\nu} \mathrm{d} \tau
$$

The measure is computed by integrating the M-LCD deviation over all possible locations $\boldsymbol{\nu} \in \mathbb{S}^{1} \times \mathbb{R}^{2}$ and dispersions $\tau$ of the kernel defined in (7). The weighting function $\mathcal{W}(\tau)$ controls the contribution of the M-LCD deviation w.r.t. the dispersion $\tau$. Minimizing such a distance measure essentially denotes optimally maintaining the probability mass of the target Dirac mixture on the manifold. The approximating samples can thereby be obtained by solving the following optimization problem under the constraint of the manifold structure, namely

$$
\mathbb{X}^{*}=\arg \min _{\mathbb{X}} \mathcal{D}(\mathbb{X}), \quad \mathbb{X}=\left\{\mathbf{x}_{i}\right\}_{i=1}^{n} \subset \mathbb{S}^{1} \times \mathbb{R}^{2}
$$


Computation of $\mathcal{D}(\mathbb{X})$ boils down to the aggregation of three integrals, i.e., $\mathcal{D}(\mathbb{X})=\mathcal{D}_{1}-2 \mathcal{D}_{2}+\mathcal{D}_{3}$, with

$$
\begin{aligned}
& \mathcal{D}_{1}=\int_{\mathbb{R}_{+}} \mathcal{W}(\tau) \int_{\mathbb{S}^{1} \times \mathbb{R}^{2}} \mathcal{F}_{\mathbb{X}}(\boldsymbol{\nu}, \tau)^{2} \mathrm{~d} \boldsymbol{\nu} \mathrm{d} \tau \\
& \mathcal{D}_{2}=\int_{\mathbb{R}_{+}} \mathcal{W}(\tau) \int_{\mathbb{S}^{1} \times \mathbb{R}^{2}} \mathcal{F}_{\mathbb{X}}(\boldsymbol{\nu}, \tau) \mathcal{F}_{\tilde{\mathbb{X}}}(\boldsymbol{\nu}, \tau) \mathrm{d} \boldsymbol{\nu} \mathrm{d} \tau, \\
& \mathcal{D}_{3}=\int_{\mathbb{R}_{+}} \mathcal{W}(\tau) \int_{\mathbb{S}^{1} \times \mathbb{R}^{2}} \mathcal{F}_{\tilde{\mathbb{X}}}(\boldsymbol{\nu}, \tau)^{2} \mathrm{~d} \boldsymbol{\nu} \mathrm{d} \tau
\end{aligned}
$$

Next, we incorporate the M-LCD equations of the approximating and target samples in (5) and (6) into the formulae above. We then obtain

$$
\begin{aligned}
\mathcal{D}_{1}= & \int_{\mathbb{R}_{+}} \mathcal{W}(\tau) \\
& \cdot \int_{\mathbb{S}^{1} \times \mathbb{R}^{2}} \sum_{i=1}^{n} \sum_{j=1}^{n} \omega_{i} \omega_{j} \kappa\left(\mathbf{x}_{i} ; \boldsymbol{\nu}, \tau\right) \kappa\left(\mathbf{x}_{j} ; \boldsymbol{\nu}, \tau\right) \mathrm{d} \boldsymbol{\nu} \mathrm{d} \tau, \\
\mathcal{D}_{2}= & \int_{\mathbb{R}_{+}} \mathcal{W}(\tau) \\
& \cdot \int_{\mathbb{S}^{1} \times \mathbb{R}^{2}} \sum_{i=1}^{n} \sum_{j=1}^{m} \omega_{i} \tilde{\omega}_{j} \kappa\left(\mathbf{x}_{i} ; \boldsymbol{\nu}, \tau\right) \kappa\left(\tilde{\mathbf{x}}_{j} ; \boldsymbol{\nu}, \tau\right) \mathrm{d} \boldsymbol{\nu} \mathrm{d} \tau, \\
\mathcal{D}_{3}= & \int_{\mathbb{R}_{+}} \mathcal{W}(\tau) \\
& \cdot \int_{\mathbb{S}^{1} \times \mathbb{R}^{2}} \sum_{i=1}^{m} \sum_{j=1}^{m} \tilde{\omega}_{i} \tilde{\omega}_{j} \kappa\left(\tilde{\mathbf{x}}_{i} ; \boldsymbol{\nu}, \tau\right) \kappa\left(\tilde{\mathbf{x}}_{j} ; \boldsymbol{\nu}, \tau\right) \mathrm{d} \boldsymbol{\nu} \mathrm{d} \tau .
\end{aligned}
$$

The double integration can be first applied in a sample-wise manner, yielding

$$
\begin{aligned}
\mathcal{D}_{1}= & \sum_{i=1}^{n} \sum_{j=1}^{n} \omega_{i} \omega_{j} \int_{\mathbb{R}_{+}} \mathcal{W}(\tau) \\
& \cdot \int_{\mathbb{S}^{1} \times \mathbb{R}^{2}} \kappa\left(\mathbf{x}_{i} ; \boldsymbol{\nu}, \tau\right) \kappa\left(\mathbf{x}_{j} ; \boldsymbol{\nu}, \tau\right) \mathrm{d} \boldsymbol{\nu} \mathrm{d} \tau, \\
\mathcal{D}_{2}= & \sum_{i=1}^{n} \sum_{j=1}^{m} \omega_{i} \tilde{\omega}_{j} \int_{\mathbb{R}_{+}} \mathcal{W}(\tau) \\
& \cdot \int_{\mathbb{S}^{1} \times \mathbb{R}^{2}} \kappa\left(\mathbf{x}_{i} ; \boldsymbol{\nu}, \tau\right) \kappa\left(\tilde{\mathbf{x}}_{j} ; \boldsymbol{\nu}, \tau\right) \mathrm{d} \boldsymbol{\nu} \mathrm{d} \tau, \\
\mathcal{D}_{3}= & \sum_{i=1}^{m} \sum_{j=1}^{m} \tilde{\omega}_{i} \tilde{\omega}_{j} \int_{\mathbb{R}_{+}} \mathcal{W}(\tau) \\
& \cdot \int_{\mathbb{S}^{1} \times \mathbb{R}^{2}} \kappa\left(\tilde{\mathbf{x}}_{i} ; \boldsymbol{\nu}, \tau\right) \kappa\left(\tilde{\mathbf{x}}_{j} ; \boldsymbol{\nu}, \tau\right) \mathrm{d} \boldsymbol{\nu} \mathrm{d} \tau .
\end{aligned}
$$

The individual distance components above share the same integration over the kernel parameters. The general form of the inner-layer integral can be formulated as

$$
\mathcal{A}(\mathbf{x}, \mathbf{y} ; \tau)=\int_{\mathbb{S}^{1} \times \mathbb{R}^{2}} \kappa(\mathbf{x} ; \boldsymbol{\nu}, \tau) \kappa(\mathbf{y} ; \boldsymbol{\nu}, \tau) \mathrm{d} \boldsymbol{\nu},
$$

with the product of kernel values being integrated over all kernel locations on the manifold $\mathbb{S}^{1} \times \mathbb{R}^{2}$. The kernel function in (7) is separable for the real and dual part, we thereby decompose the whole integral into $\mathcal{A}(\mathbf{x}, \mathbf{y}, \tau)=$ $\mathcal{A}_{\mathrm{r}}\left(\mathbf{x}_{\mathrm{r}}, \mathbf{y}_{\mathrm{r}}, \tau\right) \mathcal{A}_{\mathrm{s}}\left(\mathbf{x}_{\mathrm{s}}, \mathbf{y}_{\mathrm{s}}, \tau\right)$ and obtain

$$
\begin{aligned}
\mathcal{A}_{\mathrm{r}}\left(\mathbf{x}_{\mathrm{r}}, \mathbf{y}_{\mathrm{r}}, \tau\right) & =\int_{\mathbb{S}^{1}} \kappa_{\mathrm{r}}\left(\mathbf{x}_{\mathrm{r}} ; \boldsymbol{\nu}_{\mathrm{r}}, \tau\right) \kappa_{\mathrm{r}}\left(\mathbf{y}_{\mathrm{r}} ; \boldsymbol{\nu}_{\mathrm{r}}, \tau\right) \mathrm{d} \boldsymbol{\nu}_{\mathrm{r}} \\
& =2 \pi \mathcal{I}_{0}\left(\tau \sqrt{2+2 \mathbf{x}_{\mathrm{r}}^{\top} \mathbf{y}_{\mathrm{r}}}\right) \\
\mathcal{A}_{\mathrm{s}}\left(\mathbf{x}_{\mathrm{s}}, \mathbf{y}_{\mathrm{s}}, \tau\right) & =\int_{\mathbb{R}^{2}} \kappa_{\mathrm{s}}\left(\mathbf{y}_{\mathrm{s}} ; \boldsymbol{\nu}_{\mathrm{s}}, \tau\right) \kappa_{\mathrm{s}}\left(\mathbf{x}_{\mathrm{s}} ; \boldsymbol{\nu}_{\mathrm{s}}, \tau\right) \mathrm{d} \boldsymbol{\nu}_{\mathrm{s}} \\
& =\frac{\pi}{2 \tau} \exp \left(-\frac{\tau}{2}\left(\mathbf{x}_{\mathrm{s}}-\mathbf{y}_{\mathrm{s}}\right)^{\top}\left(\mathbf{x}_{\mathrm{s}}-\mathbf{y}_{\mathrm{s}}\right)\right) .
\end{aligned}
$$

Here, $\mathcal{I}_{0}$ denotes the modified Bessel function of the first kind of order zero. A detailed derivation can be found in Appendix A. For conciseness, we further substitute the metric for the real part on the circular domain and the dual part in the Euclidean space as $\zeta_{\mathbf{x y}}=\mathbf{x}_{\mathrm{r}}^{\top} \mathbf{y}_{\mathrm{r}}$ and $\xi_{\mathbf{x y}}=\left(\mathbf{x}_{\mathrm{s}}-\mathbf{y}_{\mathrm{s}}\right)^{\top}\left(\mathbf{x}_{\mathrm{s}}-\mathbf{y}_{\mathrm{s}}\right)$, respectively. Then, we have

$$
\mathcal{A}(\mathbf{x}, \mathbf{y}, \tau)=\frac{\pi^{2}}{\tau} \mathcal{I}_{0}\left(\tau \sqrt{2+2 \zeta_{\mathbf{x y}}}\right) \exp \left(-\frac{\tau}{2} \xi_{\mathbf{x y}}\right) .
$$

Further, the integral over the dispersion $\tau$ is given by

$$
\mathcal{B}(\mathbf{x}, \mathbf{y})=\int_{\mathbb{R}_{+}} \mathcal{W}(\tau) \mathcal{A}(\mathbf{x}, \mathbf{y} ; \tau) \mathrm{d} \tau
$$

As discussed in [21], the weighting function $\mathcal{W}(\tau)$ controls the impact of the integrated kernel values on the distance measure w.r.t. the dispersion. In theory, there are many possible functions for $\mathcal{W}(\tau)$. In practice, however, it is appealing to introduce a carefully crafted weighting function such that a closed-form solution of $\mathcal{B}(\mathbf{x}, \mathbf{y})$ can be obtained. The optimization-based sample reduction procedure can then benefit therefrom regarding convergence efficiency. Thus, we propose the following weighting function

$$
\mathcal{W}(\tau)=\tau \exp (-5 \tau / 2)
$$

The integral in (10) can then be derived as

$$
\mathcal{B}(\mathbf{x}, \mathbf{y})=\frac{2 \pi^{2}}{\left(17-8 \zeta_{\mathbf{x y}}+10 \xi_{\mathbf{x y}}+\xi_{\mathbf{x y}}^{2}\right)^{1 / 2}} .
$$

We further substitute the denominator with $\mathcal{J}(\mathbf{x}, \mathbf{y})$ such that $\mathcal{B}(\mathbf{x}, \mathbf{y})=2 \pi^{2} / \mathcal{J}(\mathbf{x}, \mathbf{y})$. A detailed derivation of the integral is given in Appendix B. The each individual component of the distance measure in (9) can be computed as

$$
\begin{aligned}
& \mathcal{D}_{1}=2 \pi^{2} \sum_{i=1}^{n} \sum_{j=1}^{n} \omega_{i} \omega_{j} \mathcal{J}\left(\mathbf{x}_{i}, \mathbf{x}_{j}\right)^{-1}, \\
& \mathcal{D}_{2}=2 \pi^{2} \sum_{i=1}^{n} \sum_{j=1}^{m} \omega_{i} \tilde{\omega}_{j} \mathcal{J}\left(\mathbf{x}_{i}, \tilde{\mathbf{x}}_{j}\right)^{-1}, \\
& \mathcal{D}_{3}=2 \pi^{2} \sum_{i=1}^{m} \sum_{j=1}^{m} \tilde{\omega}_{i} \tilde{\omega}_{j} \mathcal{J}\left(\tilde{\mathbf{x}}_{j}, \tilde{\mathbf{x}}_{j}\right)^{-1} .
\end{aligned}
$$

As stated previously, the overall distance measure is then obtained with $\mathcal{D}(\mathbb{X})=\mathcal{D}_{1}-2 \mathcal{D}_{2}+\mathcal{D}_{3}$. 


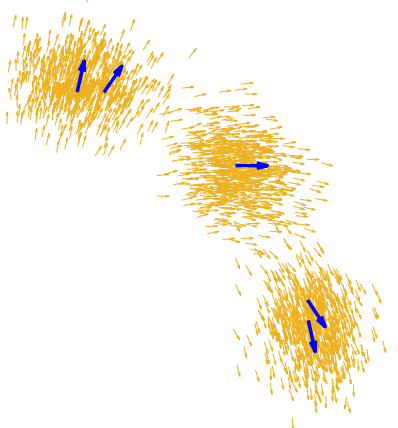

(a) $n=5, m=2000$

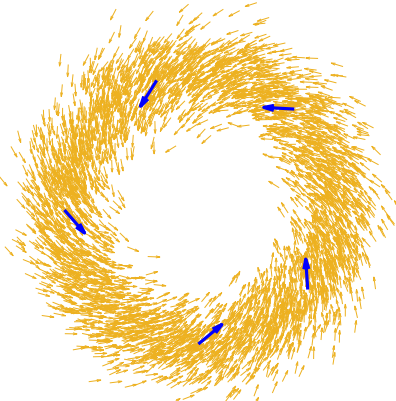

(e) $n=5, m=4000$

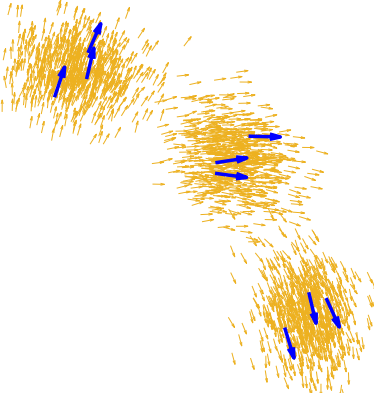

(b) $n=9, m=2000$

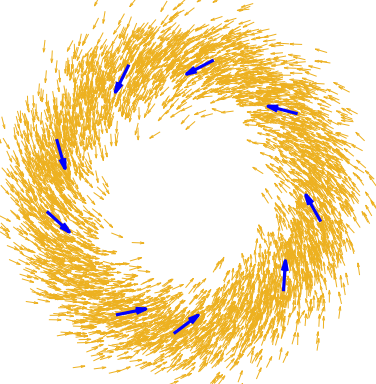

(f) $n=9, m=4000$
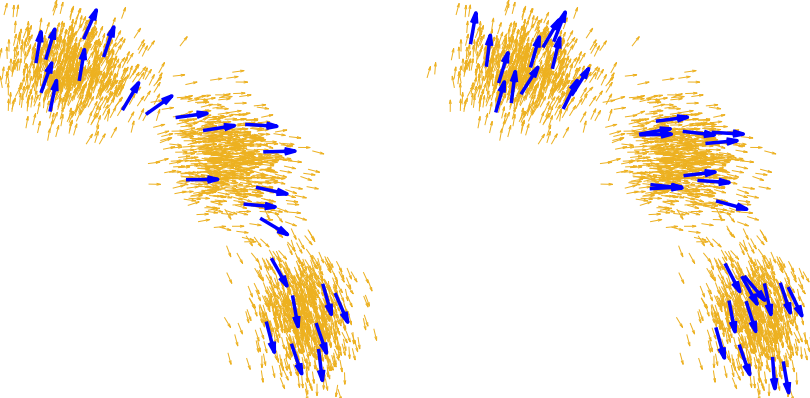

(c) $n=25, m=2000$

(d) $n=35, m=2000$

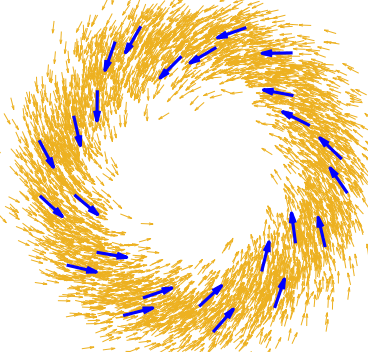

(g) $n=25, m=4000$

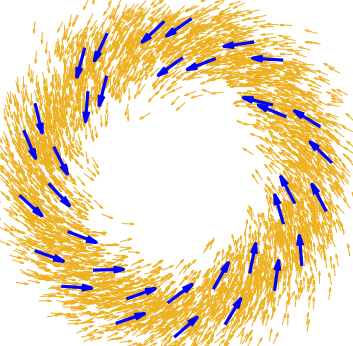

(h) $n=35, m=4000$

Figure 2: Reduction of equally weighted planar dual quaternion samples using the proposed approach. To visualize the poses, the planar dual quaternion samples are shown as arrows. Random poses (yellow, quantity denoted by $m$ ) are approximated by fewer deterministic samples (blue, quantity denoted by $n$ ). The proposed approach works reliably for different underlying distributions with various reduction scales.

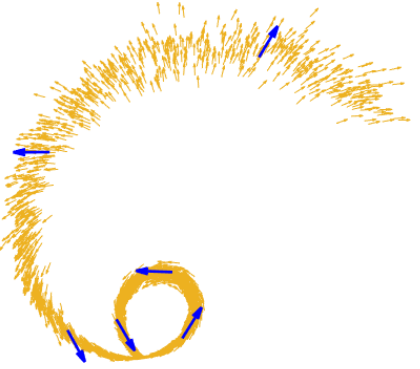

(a) $n=6, m=2000$

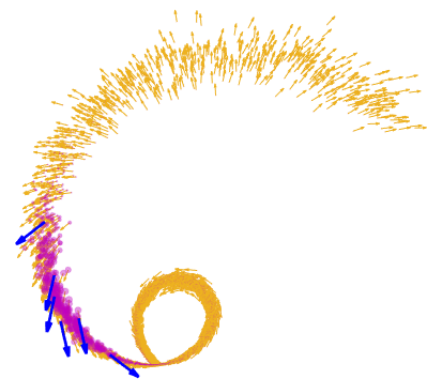

(b) $n=6, m=2000$

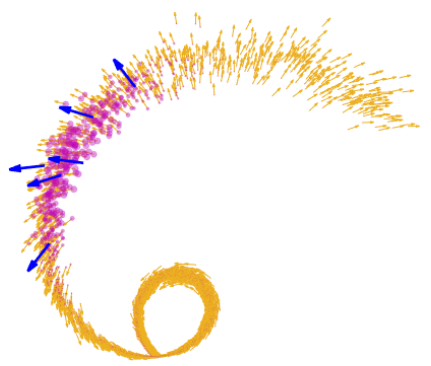

(c) $n=6, m=2000$

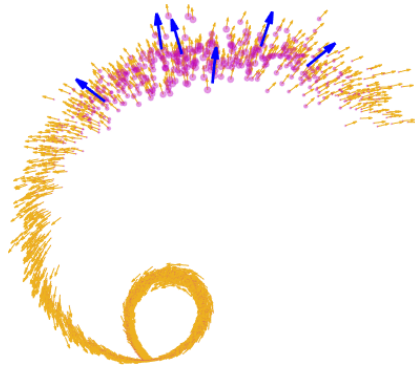

(d) $n=6, m=2000$

Figure 3: Reduction of the same target planar dual quaternion samples with varying nonuniform weights. (a) shows the reduction result of equally weighted target samples. In (b)-(c), we shift the sample weights (indicated by the size of the purple disk) clockwise. The proposed reduction scheme gives effective approximation results in all cases.

\section{Optimal Reduction via Riemannian Optimization}

As pointed out in Sec. II, the manifold of planar dual quaternions is the product of unit circle $\mathbb{S}^{1}$ and two-dimensional Euclidean space $\mathbb{R}^{2}$. During the reduction, samples are thereby confined to the Riemannian manifold $\mathbb{S}^{1} \times \mathbb{R}^{2}$. Instead of treating the approximation task formulated in (8) as a constrained optimization problem and applying classic approaches, e.g., the Lagrange multiplier, we utilize the Riemannian optimization scheme [25]. It is geometry-aware and shows improved convergence behavior provided that the manifold structure is well investigated. The derived distance measure in (13) is smooth and twice continuously differentiable. Thus, we apply the Riemannian trust-region method given in [26]. Each iterative step is first computed on the tangent plane and then retracted back to $\mathbb{S}^{1} \times \mathbb{R}^{2}$ to preserve the manifold structure. For that, the gradient of the objective function in the ambient space is essential, which is provided in Appendix C.

By minimizing the proposed distance measure, the probability mass of the random samples is optimally preserved while considering the geometric structure of the planar dual quaternion manifold. We show a few representative examples in Fig. 2 and Fig. 3 to illustrate the proposed pose reduction 


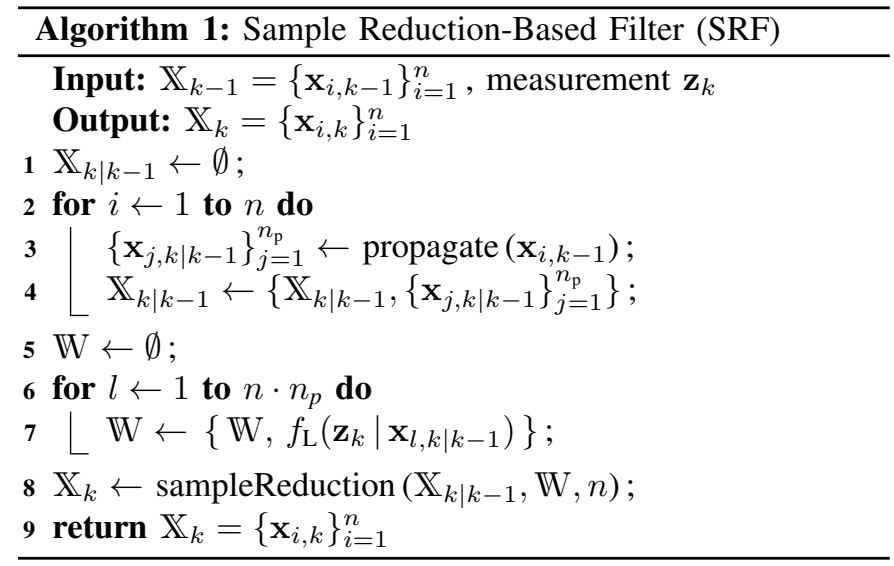

technique. For target pose samples of various randomness as well as weights, the approach works reliably for different reduction scales. It can be also observed in the figures that the approach can preserve the correlation between the real and dual part of the target samples.

\section{SAmple Reduction-Based SE(2) Estimation}

The proposed approximation approach is then integrated into a sample-based Bayesian filtering scheme for planar motion estimation. The system model is formulated as

$$
\mathbf{x}_{k}=a\left(\mathbf{x}_{k-1}, \mathbf{w}_{k-1}\right),
$$

with $\mathbf{x}_{k-1}, \mathbf{x}_{k} \in \mathbb{S}^{1} \times \mathbb{R}^{2}$ being the system state and $\mathbf{w}_{k-1}$ the system noise of domain W. $a: \mathbb{S}^{1} \times \mathbb{R}^{2} \times \mathbb{W} \rightarrow \mathbb{S}^{1} \times \mathbb{R}^{2}$ is the transition function. The measure model is given as

$$
\mathbf{z}_{k}=h\left(\mathbf{x}_{k}, \mathbf{v}_{k}\right),
$$

with $\mathbf{z}_{k} \in \mathbb{Z}$ and $\mathbf{v}_{k} \in \mathbb{V}$ representing the measurement and measurement noise, respectively. Further, $h: \mathbb{S}^{1} \times \mathbb{R}^{2} \times \mathbb{V} \rightarrow$ $\mathbb{Z}$ denotes the observation function. Planar dual quaternion samples of uniform weight are exploited as a non-parametric representation of the posterior distribution.

As shown in Alg. 1, the set of $n$ equally weighted samples $\mathbb{X}_{k-1}$ representing the posterior at time step $k-1$ is fed through the system dynamics in (14). Each planar dual quaternion sample $\mathbf{x}_{k-1}$ is propagated by $n_{\mathrm{p}}$ random samples from the system noise distribution (Alg. 1, line 1-4). A sample set $\mathbb{X}_{k \mid k-1}$ of size $n \cdot n_{\mathrm{p}}$ is thereby obtained to represent the prior distribution. Then, each prior sample is reweighted according to the likelihood $f_{\mathrm{L}}\left(\mathbf{z}_{k} \mid \mathbf{x}_{l, k \mid k-1}\right)$ derived from (15) given the measurement $\mathbf{z}_{k}$ (Alg. 1, line 5-7). The prior sample set $\mathbb{X}_{k \mid k-1}$ with nonuniform weights can then be approximated using the proposed sample reduction approach (Alg. 1, line 8). The obtained samples $\left\{\mathbf{x}_{i, k}\right\}_{i=1}^{n}$ are again equally weighted and deterministic, representing the posterior distribution of the current time step.

\section{Evaluation}

The proposed sample reduction-based planar dual quaternion filter is then evaluated in the following nonlinear $\operatorname{SE}(2)$

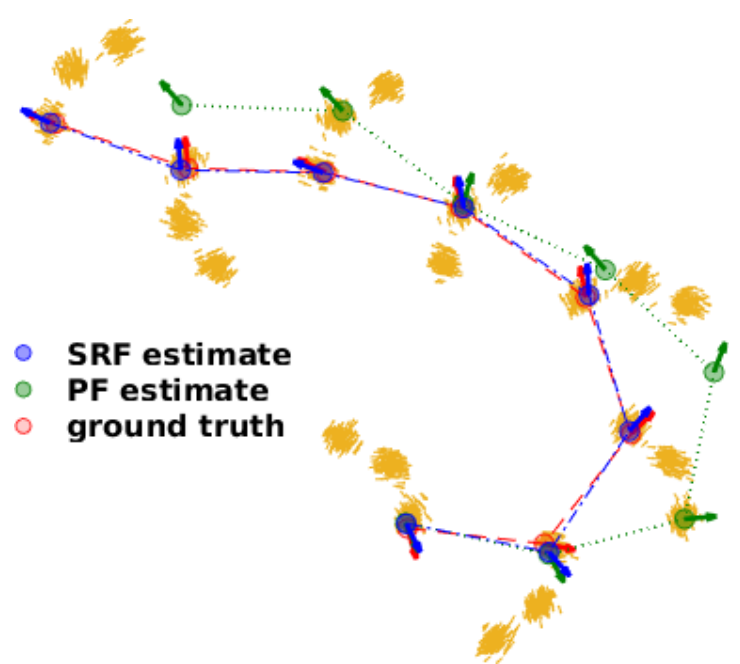

Figure 4: Exemplary trajectory segment of the evaluation run.

estimation task. The system model is $\mathbf{x}_{k}=\mathbf{x}_{k} \boxplus \mathbf{w}_{k}^{2}$, with $\mathbf{x}_{k}, \mathbf{w}_{k} \in \mathbb{S}^{1} \times \mathbb{R}^{2}$ denoting the system state and system noise, respectively. $\mathbf{w}_{k}$ is synthesized by composing a von Mises mixture-distributed rotation angle and a Gaussian-distributed translation according to (1). $\mathbf{w}_{k}^{2}:=\mathbf{w}_{k} \boxplus \mathbf{w}_{k}$ is defined as the squared system noise and has a multimodal distribution, as evident from the yellow arrows in Fig. 2 (a). Further, the measurement model is given as $\mathbf{z}_{k}=\left(\mathbf{x}_{k} \boxplus\left[1,0, \mathbf{z}_{0}^{\top}\right]^{\top} \boxplus\right.$ $\left.\mathbf{x}_{k}^{\odot}\right)_{3: 4}+\mathbf{v}_{k}$ according to (2). It measures the position of a point transformed by the planar dual quaternion state $\mathbf{x}_{k}$ from its initial location $\mathbf{z}_{0}$. The additive measurement noise $\mathbf{v}_{k}$ is assumed to follow a zero-mean Gaussian distribution.

We compare the sample reduction-based filter (SRF) proposed in Sec. IV with a plain particle filter (PF) and the SE(2)Bingham filter (SE2BF) with a progressive update step [14], [27]. As the SE2BF relies on the $\mathrm{SE}(2)$-Bingham distribution [12], we exploit $10^{5}$ random samples for fitting the parametric model offline. Moreover, 100 random samples are used by the PF to model the estimate, whereas the proposed SRF uses 5 deterministic samples via the optimal approximation. The evaluation is performed for 100 Monte Carlo runs with 10 time steps each. To quantify the tracking accuracy, we compute the orientation and position error w.r.t. the quaternion arc length and the Euclidean distance, respectively.

As shown in Fig. 5, the sample reduction-based filter gives more accurate tracking results than the particle filter. Considering that the SRF deploys much fewer samples than the PF, this shows that the proposed approximation method is effective and improves the sample efficiency considerably compared with the Monte Carlo scheme. The SE2BF fails for all runs because the parametric modeling of the uncertainty is largely violated by the multimodal noise and strong nonlinearity. We further show an exemplary run of the tracking result in Fig. 4. At each time step, we sample the system noise to propagate the state for illustrating the uncertain dynamics (depicted by yellow arrows). Though much more samples are used by the $\mathrm{PF}$, its estimates are still prone to be at wrong modes as the 


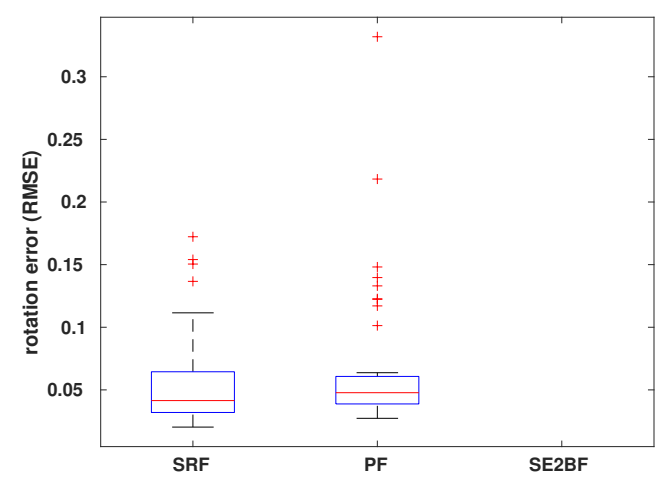

(a) orientation accuracy

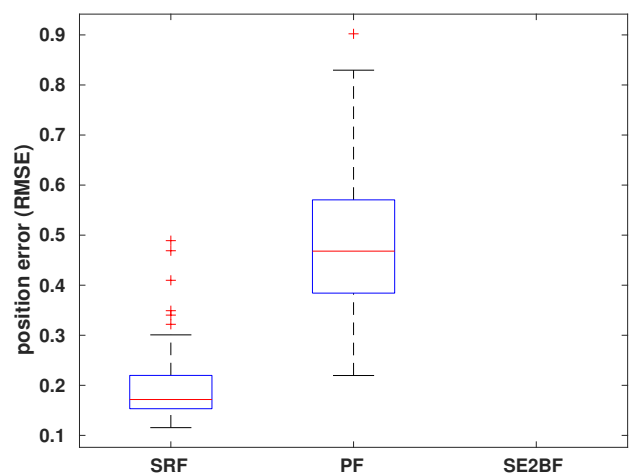

(b) position accuracy

Figure 5: Results of the simulated evaluation. The proposed sample reduction-based filter gives more accurate tracking results than the particle filter. The SE(2)-Bingham filter totally fails due to its parametric modeling of estimates.

random samples are less representative than the deterministic ones given by the optimal approximation.

\section{CONCLUSION}

In this paper, a novel sample reduction scheme is proposed for random planar dual quaternion samples by means of Dirac mixture approximation. A geometry-aware distance measure is proposed on the manifold of planar dual quaternions, based on which the new Dirac supports are obtained to preserve the probability mass via Riemannian optimization. Furthermore, we propose a sample reduction-based planar dual quaternion filter for $\mathrm{SE}(2)$ estimation. It performs better than the plain particle filter as well as the state-of-the-art dual quaternion filter using the parametric model.

There still remains much potential to exploit from the proposed methods. The current reduction approach can be further extended to the manifold of unit dual quaternions that represent spatial poses belonging to the $\mathrm{SE}(3)$ group [9]. Applying the sample-based approximation scheme to system identification and control-related tasks is also of great value. Moreover, extensive applications of the proposed filter can be done for real-world scenarios such as visual odometry and scene registration [9], [16].

\section{APPENDIX}

\section{A. Integral over the Kernel Location}

The integral of the von Mises kernel product over $\mathbb{S}^{1}$ is

$$
\begin{aligned}
\mathcal{A}_{\mathrm{r}}\left(\mathbf{x}_{\mathrm{r}}, \mathbf{y}_{\mathrm{r}}, \tau\right) & =\int_{\mathbb{S}^{1}} \kappa_{\mathrm{r}}\left(\mathbf{x}_{\mathrm{r}} ; \boldsymbol{\nu}_{\mathrm{r}}, \tau\right) \kappa_{\mathrm{r}}\left(\mathbf{y}_{\mathrm{r}} ; \boldsymbol{\nu}_{\mathrm{r}}, \tau\right) \mathrm{d} \boldsymbol{\nu}_{\mathrm{r}} \\
& =\int_{\mathbb{S}^{1}} \exp \left(\tau \boldsymbol{\nu}_{\mathrm{r}}^{\top}\left(\mathbf{x}_{\mathrm{r}}+\mathbf{y}_{\mathrm{r}}\right)\right) \mathrm{d} \boldsymbol{\nu}_{\mathrm{r}}
\end{aligned}
$$

By normalizing $\mathbf{x}_{\mathrm{r}}+\mathbf{y}_{\mathrm{r}}=\left\|\mathbf{x}_{\mathrm{r}}+\mathbf{y}_{\mathrm{r}}\right\| \widehat{\mathbf{x}_{\mathrm{r}}+\mathbf{y}_{\mathrm{r}}}$, the integral can be obtained as the normalization constant of a scaled von Mises density, i.e.,

$$
\begin{aligned}
\mathcal{A}_{\mathrm{r}}\left(\mathbf{x}_{\mathrm{r}}, \mathbf{y}_{\mathrm{r}}, \tau\right) & =\int_{\mathbb{S}^{1}} \exp \left(\tau\left\|\mathbf{x}_{\mathrm{r}}+\mathbf{y}_{\mathrm{r}}\right\| \boldsymbol{\nu}_{\mathrm{r}}^{\top} \widehat{\mathbf{x}_{\mathrm{r}}+\mathbf{y}_{\mathrm{r}}}\right) \mathrm{d} \boldsymbol{\nu}_{\mathrm{r}} \\
& =2 \pi \mathcal{I}_{0}\left(\tau\left\|\mathbf{x}_{\mathrm{r}}+\mathbf{y}_{\mathrm{r}}\right\|\right)
\end{aligned}
$$

with $\mathcal{I}_{0}$ being the zero-order modified Bessel function of the first kind. Therefore, the integral of the kernel product over $\mathbb{S}^{1}$ only depends on the geodesic arc length between the two points in consideration and we obtain $\mathcal{A}(\mathbf{x}, \mathbf{y}, \tau)=$ $2 \pi \mathcal{I}_{0}\left(\tau \sqrt{2+2 \mathbf{x}_{\mathrm{r}}^{\top} \mathbf{y}_{\mathrm{r}}}\right)$

The integral of the kernel over the two-dimensional Euclidean space for the dual part can be derived by applying the Gaussian identity given in [28, Appendix D], yielding

$$
\begin{aligned}
& \mathcal{A}_{\mathrm{s}}\left(\mathbf{x}_{\mathrm{s}}, \mathbf{y}_{\mathrm{s}}, \tau\right)=\int_{\mathbb{R}^{2}} \kappa_{\mathrm{s}}\left(\mathbf{x}_{\mathrm{s}} ; \boldsymbol{\nu}_{\mathrm{s}}, \tau\right) \kappa_{\mathrm{s}}\left(\mathbf{y}_{\mathrm{s}} ; \boldsymbol{\nu}_{\mathrm{s}}, \tau\right) \mathrm{d} \boldsymbol{\nu}_{\mathrm{s}} \\
& =\frac{\pi^{2}}{\tau^{2}} \int_{\mathbb{R}^{2}} f_{\mathcal{N}}\left(\mathbf{x}_{\mathrm{s}} ; \boldsymbol{\nu}_{\mathrm{s}}, \frac{1}{2 \tau} \mathbf{I}_{2 \times 2}\right) f_{\mathcal{N}}\left(\mathbf{y}_{\mathrm{s}} ; \boldsymbol{\nu}_{\mathrm{s}}, \frac{1}{2 \tau} \mathbf{I}_{2 \times 2}\right) \mathrm{d} \boldsymbol{\nu}_{\mathrm{s}} \\
& =\frac{\pi^{2}}{\tau^{2}} f_{\mathcal{N}}\left(\mathbf{y}_{\mathrm{s}} ; \mathbf{x}_{\mathrm{s}}, \frac{1}{\tau} \mathbf{I}_{2 \times 2}\right) \int_{\mathbb{R}^{2}} f_{\mathcal{N}}\left(\boldsymbol{\nu}_{\mathrm{s}}\right) \mathrm{d} \boldsymbol{\nu}_{\mathrm{s}} \\
& =\frac{\pi}{2 \tau} \exp \left(-\frac{\tau}{2}\left(\mathbf{x}_{\mathrm{s}}-\mathbf{y}_{\mathrm{s}}\right)^{\top}\left(\mathbf{x}_{\mathrm{s}}-\mathbf{y}_{\mathrm{s}}\right)\right) .
\end{aligned}
$$

\section{B. Integral over the Kernel Dispersion}

Given two planar dual quaternions $\mathbf{x}, \mathbf{y} \in \mathbb{S}^{1} \times \mathbb{R}^{2}$ and the weighting function in (11), the integral of the weighted $\mathcal{A}(\mathbf{x}, \mathbf{y}, \tau)$ over the kernel dispersion $\tau$ is given by

$\mathcal{B}(\mathbf{x}, \mathbf{y})=\pi^{2} \int_{\mathbb{R}^{+}} \exp \left(-\frac{\tau}{2}\left(5+\xi_{\mathbf{x y}}\right)\right) \mathcal{I}_{0}\left(\tau \sqrt{2+2 \zeta_{\mathbf{x y}}}\right) \mathrm{d} \tau$.

As stated in [29, Sec. 6.611], the following closed-form integral holds for zero-order Bessel function of the first kind

$$
\int_{\mathbb{R}_{+}} \exp (-\alpha \tau) \mathcal{I}_{0}(\beta \tau)=1 / \sqrt{\alpha^{2}-\beta^{2}},
$$

for $\forall \alpha, \beta \in \mathbb{R}_{+}, \alpha>\beta$. When setting $\alpha=0.5\left(5+\xi_{\mathbf{x y}}\right)$ and $\beta=\left(2+2 \zeta_{\mathbf{x y}}\right)^{1 / 2}$, it can be verified that $\beta \in[0,2]$ and $\alpha \in[2.5, \infty)$, therefore $\alpha>\beta$. We then have

$$
\begin{aligned}
\mathcal{B}(\mathbf{x}, \mathbf{y}) & =\frac{\pi^{2}}{\sqrt{0.25\left(5+\xi_{\mathbf{x y}}\right)^{2}-\left(2+2 \zeta_{\mathbf{x y}}\right)}} \\
& =\frac{2 \pi^{2}}{\sqrt{17-8 \zeta_{\mathbf{x y}}+10 \xi_{\mathbf{x y}}+\xi_{\mathbf{x y}}^{2}}} .
\end{aligned}
$$




\section{Derivative of the Proposed Distance Measure}

Derivatives of the point-wise distance measure in (12) w.r.t. $\mathbf{x}=\left[\mathbf{x}_{\mathrm{r}}^{\top}, \mathbf{x}_{\mathrm{s}}^{\top}\right]^{\top}$ can be obtained by applying the chain rule as

$$
\begin{aligned}
\frac{\mathrm{d} \mathcal{B}}{\mathrm{d} \mathbf{x}_{\mathrm{r}}} & =\frac{\mathrm{d} \mathcal{B}}{\mathrm{d} \zeta_{\mathbf{x y}}} \frac{\mathrm{d} \zeta_{\mathbf{x y}}}{\mathrm{d} \mathbf{x}_{\mathrm{r}}}=\frac{8 \pi^{2}}{\mathcal{J}(\mathbf{x}, \mathbf{y})^{3}} \mathbf{y}_{\mathbf{r}}, \\
\frac{\mathrm{d} \mathcal{B}}{\mathrm{d} \mathbf{x}_{\mathrm{s}}} & =\frac{\mathrm{d} \mathcal{B}}{\mathrm{d} \xi_{\mathbf{x y}}} \frac{\mathrm{d} \xi_{\mathbf{x y}}}{\mathrm{d} \mathbf{x}_{\mathrm{s}}}=-4 \pi^{2} \frac{5+\xi_{\mathbf{x y}}}{\mathcal{J}(\mathbf{x}, \mathbf{y})^{3}}\left(\mathbf{x}_{\mathrm{s}}-\mathbf{y}_{\mathrm{s}}\right) .
\end{aligned}
$$

Thus, the derivatives of the distance measure components in (13) w.r.t. the approximating sample locations $\mathbf{x}_{i}$ in $\mathbb{X}$ can be derived. For instance, we have $\frac{\mathrm{d} \mathcal{D}_{1}}{\mathrm{~d} \mathbf{x}_{i}}=\left[\frac{\mathrm{d} \mathcal{D}_{1}^{\top}}{\mathrm{d} \mathbf{x}_{i, \mathrm{r}}}, \frac{\mathrm{d} \mathcal{D}_{1}^{\top}}{\mathrm{d} \mathbf{x}_{i, \mathrm{~s}}}\right]^{\top}$, with

$$
\begin{aligned}
\frac{\mathrm{d} \mathcal{D}_{1}}{\mathrm{~d} \mathbf{x}_{i, \mathrm{r}}} & =16 \pi^{2} \omega_{i} \sum_{j=1}^{n} \frac{\omega_{j}}{\mathcal{J}\left(\mathbf{x}_{i}, \mathbf{x}_{j}\right)^{3}} \mathbf{x}_{j, \mathrm{r}}, \\
\frac{\mathrm{d} \mathcal{D}_{1}}{\mathrm{~d} \mathbf{x}_{i, \mathrm{~s}}} & =-8 \pi^{2} \omega_{i} \sum_{j=1}^{n} \omega_{j} \frac{5+\xi_{\mathbf{x}_{i} \mathbf{x}_{j}}}{\mathcal{J}\left(\mathbf{x}_{i}, \mathbf{x}_{j}\right)^{3}}\left(\mathbf{x}_{i, \mathrm{~s}}-\mathbf{x}_{j, \mathrm{~s}}\right) .
\end{aligned}
$$

Further, we have $\frac{\mathrm{d} \mathcal{D}_{2}}{\mathrm{~d} \mathbf{x}_{i}}=\left[\frac{\mathrm{d} \mathcal{D}_{2}^{\top}}{\mathrm{d} \mathbf{x}_{i, \mathrm{r}}}, \frac{\mathrm{d} \mathcal{D}_{2}^{\top}}{\mathrm{d} \mathbf{x}_{i, \mathrm{~s}}}\right]^{\top}$, with

$$
\begin{aligned}
\frac{\mathrm{d} \mathcal{D}_{2}}{\mathrm{~d} \mathbf{x}_{i, \mathrm{r}}} & =8 \pi^{2} \omega_{i} \sum_{j=1}^{m} \frac{\tilde{\omega}_{j}}{\mathcal{J}\left(\mathbf{x}_{i}, \tilde{\mathbf{x}}_{j}\right)^{3}} \tilde{\mathbf{x}}_{j, \mathrm{r}}, \\
\frac{\mathrm{d} \mathcal{D}_{2}}{\mathrm{~d} \mathbf{x}_{i, \mathrm{~s}}} & =-4 \pi^{2} \omega_{i} \sum_{j=1}^{m} \tilde{\omega}_{j} \frac{5+\xi_{\mathbf{x}_{i} \tilde{\mathbf{x}}_{j}}}{\mathcal{J}\left(\mathbf{x}_{i}, \tilde{\mathbf{x}}_{j}\right)^{3}}\left(\mathbf{x}_{i, \mathrm{~s}}-\tilde{\mathbf{x}}_{j, \mathrm{~s}}\right) .
\end{aligned}
$$

Then, we have $\frac{\mathrm{d} \mathcal{D}}{\mathrm{d} \mathbf{x}_{i}}=\frac{\mathrm{d} \mathcal{D}_{1}}{\mathrm{~d} \mathbf{x}_{i}}-2 \frac{\mathrm{d} \mathcal{D}_{2}}{\mathrm{~d} \mathbf{x}_{i}}$.

\section{ACKNOWLEDGMENT}

This work is supported by the German Research Foundation (DFG) under grant HA 3789/16-1.

\section{REFERENCES}

[1] S. Thrun, "Probabilistic Robotics," Communications of the ACM, vol. 45, no. 3, pp. 52-57, 2002.

[2] M. Montemerlo, "FastSLAM: A Factored Solution to the Simultaneous Localization and Mapping Problem with Unknown Data Association," Ph.D. dissertation, Carnegie Mellon University, Pittsburgh, PA, July 2003.

[3] W. Hess, D. Kohler, H. Rapp, and D. Andor, "Real-Time Loop Closure in 2D LIDAR SLAM," in Proceedings of the 2016 IEEE International Conference on Robotics and Automation (ICRA 2016), Stockholm, Sweden, May 2016, pp. 1271-1278.

[4] S. Xuan, S. Li, M. Han, X. Wan, and G. Xia, "Object Tracking in Satellite Videos by Improved Correlation Filters With Motion Estimations," IEEE Transactions on Geoscience and Remote Sensing, vol. 58, no. 2, pp. 1074-1086, 2020.

[5] S. Hare, S. Golodetz, A. Saffari, V. Vineet, M.-M. Cheng, S. L. Hicks, and P. H. Torr, "Struck: Structured Output Tracking with Kernels," IEEE Transactions on Pattern Analysis and Machine Intelligence, vol. 38, no. 10, pp. 2096-2109, 2015.

[6] C. Forster, L. Carlone, F. Dellaert, and D. Scaramuzza, "On-Manifold Preintegration for Real-Time Visual-Inertial Odometry," IEEE Transactions on Robotics, vol. 33, no. 1, pp. 1-21, 2016.

[7] M. Bloesch, M. Burri, S. Omari, M. Hutter, and R. Siegwart, "Iterated Extended Kalman Filter Based Visual-Inertial Odometry Using Direct Photometric Feedback," The International Journal of Robotics Research, vol. 36, no. 10, pp. 1053-1072, 2017.

[8] S. J. Julier and J. K. Uhlmann, "Unscented Filtering and Nonlinear Estimation," Proceedings of the IEEE, vol. 92, no. 3, pp. 401-422, 2004.

[9] S. Bultmann, K. Li, and U. D. Hanebeck, "Stereo Visual SLAM Based on Unscented Dual Quaternion Filtering," in Proceedings of the 22nd International Conference on Information Fusion (Fusion 2019), Ottawa, Canada, July 2019.
[10] I. Gilitschenski, G. Kurz, S. J. Julier, and U. D. Hanebeck, "Unscented Orientation Estimation Based on the Bingham Distribution," IEEE Transactions on Automatic Control, vol. 61, no. 1, pp. 172-177, Jan. 2016.

[11] W. Feiten and M. Lang, "MPG - A Framework for Reasoning on 6 DoF Pose Uncertainty," arXiv preprint arXiv:1707.01941, 2017.

[12] I. Gilitschenski, G. Kurz, S. J. Julier, and U. D. Hanebeck, "A New Probability Distribution for Simultaneous Representation of Uncertain Position and Orientation," in Proceedings of the 17th International Conference on Information Fusion (Fusion 2014), Salamanca, Spain, July 2014.

[13] I. Gilitschenski, G. Kurz, and U. D. Hanebeck, "A Stochastic Filter for Planar Rigid-Body Motions," in Proceedings of the 2015 IEEE International Conference on Multisensor Fusion and Integration for Intelligent Systems (MFI 2015), San Diego, California, USA, Sept. 2015.

[14] K. Li, G. Kurz, L. Bernreiter, and U. D. Hanebeck, "Nonlinear Progressive Filtering for SE(2) Estimation," in Proceedings of the 21st International Conference on Information Fusion (Fusion 2018), Cambridge, United Kingdom, July 2018.

[15] U. D. Hanebeck, K. Briechle, and A. Rauh, "Progressive Bayes: A New Framework for Nonlinear State Estimation," in Proceedings of SPIE, AeroSense Symposium, vol. 5099, Orlando, Florida, USA, May 2003, pp. $256-267$.

[16] K. Li, G. Kurz, L. Bernreiter, and U. D. Hanebeck, "Simultaneous Localization and Mapping Using a Novel Dual Quaternion Particle Filter," in Proceedings of the 21 st International Conference on Information Fusion (Fusion 2018), Cambridge, United Kingdom, July 2018.

[17] M. S. Arulampalam, S. Maskell, N. Gordon, and T. Clapp, "A Tutorial on Particle Filters for Online Nonlinear/Non-Gaussian Bayesian Tracking," IEEE Transactions on Signal Processing, vol. 50, no. 2, pp. 174-188, 2002.

[18] G. Kurz, F. Pfaff, and U. D. Hanebeck, "Discrete Recursive Bayesian Filtering on Intervals and the Unit Circle," in Proceedings of the 2016 IEEE International Conference on Multisensor Fusion and Integration for Intelligent Systems (MFI 2016), Baden-Baden, Germany, Sept. 2016.

[19] _ _ "Application of Discrete Recursive Bayesian Estimation on Intervals and the Unit Circle to Filtering on SE(2)," IEEE Transactions on Industrial Informatics, vol. 14, no. 3, pp. 1197-1206, Mar. 2018.

[20] K. Li, F. Pfaff, and U. D. Hanebeck, "Grid-Based Quaternion Filter for SO(3) Estimation," in Proceedings of the 2020 European Control Conference (ECC 2020), Saint Petersburg, Russia, May 2020.

[21] U. D. Hanebeck, "Optimal Reduction of Multivariate Dirac Mixture Densities," at - Automatisierungstechnik, Oldenbourg Verlag, vol. 63, no. 4, pp. 265-278, Apr. 2015.

[22] D. Frisch, K. Li, and U. D. Hanebeck, "Optimal Reduction of Dirac Mixture Densities on the 2-Sphere," in Proceedings of the 21st IFAC World Congress (IFAC 2020), Berlin, Germany, July 2020.

[23] K. Li, F. Pfaff, and U. D. Hanebeck, "Geometry-Driven Stochastic Modeling of SE(3) States Based on Dual Quaternion Representation," in Proceedings of the 2019 IEEE International Conference on Multisensor Fusion and Integration for Intelligent Systems (MFI 2019), Taipei, Republic of China, May 2019.

[24] U. D. Hanebeck and V. Klumpp, "Localized Cumulative Distributions and a Multivariate Generalization of the Cramér-von Mises Distance," in Proceedings of the 2008 IEEE International Conference on Multisensor Fusion and Integration for Intelligent Systems (MFI 2008), Seoul, Republic of Korea, Aug. 2008, pp. 33-39.

[25] P.-A. Absil, R. Mahony, and R. Sepulchre, Optimization Algorithms on Matrix Manifolds. Princeton University Press, 2009.

[26] N. Boumal, B. Mishra, P.-A. Absil, and R. Sepulchre, "Manopt, a Matlab Toolbox for Optimization on Manifolds," Journal of Machine Learning Research, vol. 15, pp. 1455-1459, 2014.

[27] K. Li, D. Frisch, S. Radtke, B. Noack, and U. D. Hanebeck, "Wavefront Orientation Estimation Based on Progressive Bingham Filtering," in Proceedings of the IEEE ISIF Workshop on Sensor Data Fusion: Trends, Solutions, Applications (SDF 2018), Oct. 2018.

[28] R. P. Mahler, Statistical Multisource-Multitarget Information Fusion. Artech House Norwood, MA, 2007, vol. 685.

[29] I. S. Gradshteyn and I. M. Ryzhik, Table of Integrals, Series, and Products. Academic press, 2014. 